\title{
INITIAL MOTION IN FIELDS OF FORCE*
}

\author{
BY \\ AARON FIALKOW
}

1. Introduction. The motion of a particle in an arbitrary positional field of force suggests a number of problems in the differential geometry of plane curves. Several important families of curves are associated with every field of force. We here mention the following:

1. The totality of paths along which a particle starting from rest may move under the influence of the force. These $\infty^{2}$ curves are a subset of the $\infty^{3}$ dynamical trajectories of the field of force.

2. The family of curves whose direction at each point coincides with that of the force. These curves are the $\infty^{1}$ lines of force.

3 . The system of curves on each of which the force has a constant direction. These are the $\infty^{1}$ isoclinal curves or isoclines of the field.

In this paper, we are concerned with certain geometric properties of these three families of curves. The theorems which are obtained are therefore a partial description of the photograph of the field of force.

Consider the motion of a particle which starts from rest at a point where the force is not zero. A first approximation to the path is given by the line of force on which the particle initially lies. However, the effect of inertia compels the particle to depart from this line of force and to travel in a straighter path. In general, the line of force and the trajectory will have the same initial direction but different initial curvatures. This situation and the problem of obtaining a suitable approximation to the beginning of the path were investigated by Kasner $\dagger$ and the writer $\ddagger$ in previous papers. Kasner showed that the curvature of the line of force is always three times the curvature of the trajectory. When the initial curvature of the line of force is zero, Kasner studied the ratio between the infinitesimal departures of the line of force and of the path from their common tangent. We defined the set of these limiting ratios as the ratio set. Kasner obtained a theorem, assuming that the line of force has integral order of contact with its tangent, which we generalized to apply for any finite order of contact:

* Presented to the Society, April 10, 1936; received by the editors December 4, 1935.

$\dagger$ For a complete report of Kasner's work, see Proceedings of the National Academy of Sciences, vol. 20 (1934), pp. 130-136. Some results also appear in these Transactions, 1906-1910; Bulletin of the American Mathematical Society, vol. 16 (1909-1910), p. 172; Differential Geometric Aspects of Dynamics, American Mathematical Society Colloquium Publications, vol. 3, part 2, 1913, p. 9; Science, vol. 75 (1932), p. 671; Zurich Congress Proceedings, 1932, vol. 2, p. 180.

$\ddagger$ These Transactions, vol. 38 (1935), pp. 89-105. 
THEOREM A. If the line of force has contact of order $\alpha$ with the tangent line, the trajectory will also have contact of order $\alpha$; and the ratio set will be $2 \alpha+1$.

We also discussed the case of infinite order contact as well as some cases in which no definite order of contact existed and showed that the ratio set is always a closed positive interval which depends upon the contact between the line of force and its tangent. However, in the results that appear below, as well as in Theorem A, the ratio set always reduces to a single number; that is, there is a unique limiting ratio.

In the derivation of the formula for the ratio set, a special set of axes was employed which is also useful here. The initial point is taken as the origin of coordinates and the tangent to the line of force as the $x$-axis. An equation in $x$ and $y$ in which the variables are referred to the above set of axes has been called normal. The notion of the direction function at the initial point which we previously introduced also plays an important role in the present paper. If $D(x, y)$ is the direction of the force normalized with reference to a given initial point, then $y=D(x, 0)$ is the equation of the direction function associated with that point. The right-hand member represents the value of the slope at each point of the tangent line drawn to the line of force at the initial point. We proved a lemma concerning the line of force and the direction function to which we shall refer in subsequent proofs. This lemma may be stated as follows:

LEMma. The limit of the ratio of the infinitesimal departures of the line of force and of the curve

$$
y=\int_{0}^{x} D(x, 0) d x
$$

from their common tangent is always unity.

It follows from this lemma and Theorem A that

THEOREM B. The points at which the ratio set is $2 n+1$ are identical with the points where the first $(n-1)$ derivatives* of the direction function vanish.

The previous papers of Kasner and the writer investigated the geometric relationship between certain dynamical trajectories and lines of force. This relationship was stated in theorems about the ratio set. In this paper we describe the relationship between the ratio set and two new types of curves.

At certain points the ratio set is exceptional. These points usually form

* In our previous work, we assumed the existence of continuous first partial derivatives of the components of the field of force. At present we assume the existence and continuity of all derivatives which appear in the proofs. 
a curve. We discuss some geometric properties of this curve and indicate how the ratio set depends upon the order of contact between this curve and its tangent.

The system of isoclinal curves of a field of force is geometrically determined by the lines of force. This leads to the conjecture that theorems similar to those previously derived for the lines of force apply in the case of the isoclines. The correctness of this guess is confirmed in our work. It is also shown that there is a close connection between the geometry of the system of iterated isoclinal curves and the ratio set.

2. Ratio points and ratio curves. It has been shown by Kasner that, in general, the ratio set is 3 . In what follows, we discuss certain properties of the points at which the ratio set has an exceptional value. We call a point of a positional field at which the ratio set is not 3 a ratio point. A ratio curve is defined as a curve that consists entirely of ratio points. The fundamental relationship between the ratio points and the isoclines is given by

THEOREM I. The ratio points are identical with the points at which any isoclinal curve of the field of force either is tangent to a line of force or has a singularity.

Let $D(a, b)$ be the direction of the force at a ratio point $(a, b)$. The components of the field are $\phi(x, y)$ and $\psi(x, y)$. In general, $\psi(a, b) / \phi(a, b)$ or $D(a, b)$ is not zero and a rotation and translation of axes is needed to write the equations in the normal form. In the following the subscript 1 is used to indicate normal coordinates and normal functions. The relations between the normal and given coordinates are

$$
\begin{aligned}
& x=x_{1} \cos \theta-y_{1} \sin \theta+a, \\
& y=x_{1} \sin \theta+y_{1} \cos \theta+b,
\end{aligned}
$$

where $\tan \theta=D(a, b)$. Similarly, we have

$$
\begin{aligned}
& \phi_{1}\left(x_{1}, y_{1}\right)=\phi(x, y) \cdot \cos \theta+\psi(x, y) \cdot \sin \theta, \\
& \psi_{1}\left(x_{1}, y_{1}\right)=-\phi(x, y) \cdot \sin \theta+\psi(x, y) \cdot \cos \theta,
\end{aligned}
$$

where $x$ and $y$ are to be replaced by their values given by (1). As stated in Theorem $\mathrm{B}$, the ratio set at the initial point depends upon the behavior of the direction function $\psi_{1}\left(x_{1}, 0\right) / \phi_{1}\left(x_{1}, 0\right)$ or $D_{1}\left(x_{1}, 0\right)$. From (1) and (2), it follows that $D_{1}\left(x_{1}, 0\right)$ is equivalent to

$$
\frac{\psi\left(x_{1} \cos \theta+a, x_{1} \sin \theta+b\right) \phi(a, b)-\phi\left(x_{1} \cos \theta+a, x_{1} \sin \theta+b\right) \psi(a, b)}{\psi\left(x_{1} \cos \theta+a, x_{1} \sin \theta+b\right) \psi(a, b)+\phi\left(x_{1} \cos \theta+a, x_{1} \sin \theta+b\right) \phi(a, b)} .
$$

We assume without loss of generality that $\phi(a, b) \neq 0$ and divide both numera- 
tor and denominator of the last expression by $\phi\left(x_{1} \cos \theta+a, x_{1} \sin \theta+b\right) \cdot \phi(a, b)$. Therefore

$$
D_{1}\left(x_{1}, 0\right)=\frac{D\left(x_{1} \cos \theta+a, x_{1} \sin \theta+b\right)-D(a, b)}{D\left(x_{1} \cos \theta+a, x_{1} \sin \theta+b\right) D(a, b)+1} .
$$

Since $(a, b)$ is a ratio point, by Theorem $\mathrm{B},\left(d / d x_{1}\right) D_{1}\left(x_{1}, 0\right)=0$. It follows from (3) that

$$
D_{x}(a, b)+D(a, b) \cdot D_{y}(a, b)=0 .
$$

Thus every ratio point is a solution of (4). The converse statement is also true.* $^{*}$

The equation of any isoclinal curve is $D(x, y)=c_{1}$. Differentiating, we obtain

$$
D_{x}(x, y)+y^{\prime} \cdot D_{y}(x, y)=0
$$

which is the differential equation of the isoclinal curves. If an isocline is tangent to a line of force at $\left(x_{0}, y_{0}\right)$, then $y^{\prime}=D(x, y)$. Substituting in (5) and comparing with (4), we conclude that $\left(x_{0}, y_{0}\right)$ is a ratio point. At a singularity of an isocline, $D_{x}=D_{y}=0$. From (4), it follows that such a point is also a ratio point. Since every ratio point lies on some isocline, the converse of the above statements is a consequence of the fact that $y^{\prime}=D$ and $D_{x}=D_{y}=0$ are the only simultaneous solutions of (4) and (5). This concludes the proof.

The ratio curves are the solutions of (4). In general, a single ratio curve passes through a given ratio point. For this case, we have

THEOREM II. Let a single ratio curve pass through a fixed ratio point in a direction which is not parallel to the force. Then the ratio set at this point is 5 . Conversely, if the ratio set is 5 at a point through which there passes a unique ratio curve, this curve cuts the corresponding line of force.

We suppose that the fixed ratio point is the origin and that the coordinates are normal. Differentiating (4), we obtain

$$
D_{x x}+D_{x} D_{y}+D D_{x y}+\left(D_{x y}+D_{y}^{2}+D D_{y y}\right) \cdot y^{\prime}=0 .
$$

By the choice of coordinates $D(0,0)$ is zero. Hence, by Theorem $\mathrm{B}, D_{x}(0,0)$ is also zero. Since there is a unique ratio curve through the origin, it follows that $D_{x y}+D_{y}^{2}+D D_{y y} \neq 0$ at the origin. Hence $y^{\prime}$ vanishes when and only when $D_{x x}$ is zero. By Theorem B, we find that the ratio set is 5 only if $D_{x x} \neq 0$. Since by hypothesis, $y^{\prime} \neq 0$ and the slope of the corresponding line of force vanishes, this result proves the theorem.

The analogue for ratio curves of Theorem $A$ is

\footnotetext{
* If $D_{x}+D D_{y} \equiv 0$, all the lines of force are straight lines and every point is a ratio point.
} 
THEOREM III. Let a single ratio curve pass through a fixed ratio point in the direction of the force. Let the ratio curve have contact of order $\alpha$ with its tangent at this point. Then the line of force and the trajectory through the ratio point will both have contact of order $\alpha+2$ with their common tangent; and the ratio set will be $2 \alpha+5$.

We choose the coordinate axes as in the proof of the previous theorem. 'The implicit equation of the ratio curve is (4). By the theorem of the mean, this equation may be written as

$$
\begin{aligned}
& {\left[D_{x}(x, 0)+D(x, 0) \cdot D_{y}(x, 0)\right]} \\
& +y \cdot\left[D_{x y}(x, \theta y)+D_{y}{ }^{2}(x, \theta y)+D(x, \theta y) \cdot D_{y y}(x, \theta y)\right]=0,0<\theta<1 .
\end{aligned}
$$

Representing the bracketed expressions by $E(x)$ and $F(x, y)$ respectively, the equation of the ratio curve is

$$
E(x)+y \cdot F(x, y)=0,
$$

where by hypothesis, $F(0,0) \neq 0$. Since the ratio curve has contact of order $\alpha$,

$$
\lim _{x \rightarrow 0} \frac{E(x)}{x^{\alpha+1}}=c_{2} \quad(\neq 0) .
$$

The line of force is a solution of $y^{\prime}=D(x, y)$. Differentiating this equation, and applying the theorem of the mean, we obtain

$$
y^{\prime \prime}=E(x)+y \cdot F(x, y) .
$$

Let the line of force have generalized contact* of order $\beta$. We shall show that $\beta>\alpha$. Suppose $\beta \leqq \alpha$. Choose $\gamma$ such that $\gamma+2>\beta>\gamma$. Dividing both members of (8) by $x^{\gamma+1}$ and letting $x$ approach zero, we find that $\lim _{x \rightarrow 0} y^{\prime \prime} / x^{\gamma+1}=0$. Hence, by a repeated application of L'Hospital's Rule, the line of force has generalized contact of order $\geqq \gamma+2>\beta$. This contradiction shows that $\beta>\alpha$. We divide both members of (8) by $x^{\alpha+1}$ and let $x$ approach zero. Since $\beta>\alpha$, by $(7), \lim _{x \rightarrow 0} y^{\prime \prime} / x^{\alpha+1}=c_{2}$. Therefore the line of force has ordinary contact of order $\alpha+2$; i.e., $\beta=\alpha+2$. The remainder of the theorem follows immediately from Theorem A.

* We defined generalized contact in our previous paper as follows:

A curve $y=f(x)$, where $f(x)$ is single-valued, continuous and

$$
\lim _{x \rightarrow+0} \frac{f(x)}{x}=0
$$

has generalized contact of order $\alpha$ with the $x$-axis if $\alpha$ is the upper bound of all numbers $k$ such that

$$
\lim _{x \rightarrow+0} \frac{f(x)}{x^{k+1}}=0 .
$$

$\alpha$ may be finite or infinite. 
3. Isoclinal curves. The investigation of the isoclines of the field of force leads to results similar to those obtained for lines of force and ratio curves. From the point of view of the order of contact between one of these curves and its tangent at a fixed ratio point, the isoclines occupy an intermediate position between the lines of force and the ratio curves. Thus the analogue of Theorem A and Theorem III is

THEOREM IV. Let a fixed point be a non-singular point of the isocline on which it lies. Let the isocline have contact of order $\alpha$ with the element of force at this point. Then the line of force and the trajectory through the point will both have contact of order $\alpha+1$ with their common tangent; and the ratio set will be $2 \alpha+3$.

The normal equation of the isoclinal curve is $D(x, y)=0$, or, using the theorem of the mean,

$$
D(x, 0)+y \cdot D(x, \theta y)=0, \quad 0<\theta<1 .
$$

By hypothesis, $\lim _{x \rightarrow 0} y / x^{\alpha+1}=c_{3}(\neq 0)$. Since the origin is not a singular point of the isocline, $D_{y}(0,0) \neq 0$. Therefore, by $(9), \lim _{x \rightarrow 0} D(x, 0) / x^{\alpha+1}=c_{4}(\neq 0)$. It follows from L'Hospital's Rule that the curve $y=\int_{0}^{x} D(x, 0) d x$ has contact of order $\alpha+1$ with the $x$-axis. Hence, by the lemma, the line of force must also have contact of order $\alpha+1$ with its tangent. The remainder of the theorem is a consequence of Theorem $\mathrm{A}$.

A partially equivalent theorem for integral values of $\alpha$ exists. This theorem involves the following construction: The lines of force generate a family of isoclinal curves. These isoclinal curves in turn are the base of a second family of isoclines. By this process of iteration, any number of isoclinal families may be obtained if the components of the field of force have a sufficient number of continuous partial derivatives. For this configuration of iterated isoclinal families we have

THEOREM V. Let the first $n$ iterated isoclinal curves through a fixed point be tangent to the corresponding element of force. Let the $(n+1)$ st iterated isoclinal curve pass through this point in a direction which is not parallel to the element of force. Then, if the fixed point is a non-singular point of these curves, the line of force and the trajectory through the point will both have contact of order $n+1$ with their common tangent; and the ratio set will be $2 n+3$.

This theorem is also an incomplete generalization of Theorem I. The proof follows. We assume the existence and continuity of all partial derivatives whose orders do not exceed $n+1$. The differential equation of the first family of isoclines is given by (5). Hence the second iterated isoclinal curves 
are the solutions of $D_{x}+\left(y^{\prime}\right)_{1} \cdot D_{y}=0$, where $\left(y^{\prime}\right)_{1}$ is any fixed value of $y^{\prime}$. We differentiate this equation and obtain

$$
D_{x x}+y^{\prime} \cdot D_{x_{y}}+\left(y^{\prime}\right)_{1}\left[D_{x_{y}}+y^{\prime} D_{y y}\right]=0
$$

as the differential equation of the family. By repeating this operation a sufficient number of times the differential equation of the $k$ th iterated isoclinal curves can be obtained. It is easy to show that the form of this equation is

$$
D_{x^{(k)}}+y^{\prime} \cdot D_{x^{(k-1)} y}+G=0 .
$$

$G$ is a polynomial in $y^{\prime}$ and the partial derivatives of $D(x, y)$. Each term of $G$ is multiplied by at least one $\left(y^{\prime}\right)_{i}(i<k)$, where $\left(y^{\prime}\right)_{i}$ is a fixed value of the slope of the $i$ th iterated isoclines. By hypothesis, since the coordinates are normal, the first $n$ iterated isoclinal curves have zero slope at the origin. Hence zero must be substituted for $\left(y^{\prime}\right)_{i}(i<n+1)$ in calculating the equations of the isoclines which pass through the origin. Letting $k=1,2, \cdots, n$ in (10), we find that $D_{x^{(k)}}(0,0)=0$. Similarly if $k=n+1$ in (10), $G$ vanishes at the origin. By hypothesis, $\left(y^{\prime}\right)_{n+1}$ differs from zero at the origin. Therefore if $D_{x^{(n+1)}}(0,0)=0$, the same is true for $D_{x^{(n)}}(0,0)$ and the origin is a singular point of the $(n+1)$ st isocline. This contradiction shows that $D_{x^{(n+1)}}(0,0) \neq 0$. It follows that the curve $y=\int_{0}^{x} D(x, 0) d x$ has contact of order $n+1$. The rest of the proof is similar to that of Theorem IV.

4. Generalizations. The resu ts stated in Theorems III and IV may be extended to the case in which $\alpha$ denotes generalized instead of ordinary order of contact. Theorems similar to Theorem $\mathrm{V}$ of our previous paper are obtained. If the field of force is analytic, a result analogous to Theorem III exists when more than one ratio curve pass through the fixed ratio point. In this case, it follows from Weierstrass' Preparation Theorem for two variables that only a finite number of ratio curves can intersect at the point. With certain modifications the sum of their orders of contact replaces the single order of contact in Theorem III.

The curves upon which this study of the field of force is based are the lines of force, isoclines, and ratio curves. When may a curve be a member of more than one of these classes? The answer is simple. A curve cannot belong to two of these classes without being a member of the third. The only curves. which are simultaneously lines of force, isoclines, and ratio curves are the straight lines.

Columbia University,

NEW YORK, N. Y. 